\title{
Chaos Synchronization and Parameter Identification of a New Fractional System
}

\author{
Hong-gang Dang ${ }^{1, a}$ \\ ${ }^{1}$ School of Mathematic and statistics, Tianshui Normal University, Gansu741001, China \\ ahonggangd@163.com
}

Keywords: Fractional-order, synchronization, feedback control, hyperchaotic system, adaptive

\begin{abstract}
This paper propose a novel fractional-order hyperchaotic system, the hyper-chaotic system is investigated numerically by using linear transfer function approximation. In addition, based on the stability theorem of fractional systems, adaptive feedback control method is used for synchronization of fractional-order hyperchaotic systems with unknown parameters. Meanwhile, adaptive synchronization controller and recognizing rules of the uncertain parameters are designed. The simulation example is include to confirm validity and synchronization performance of the advocated design methodology.
\end{abstract}

\section{Introduction}

Fractional calculus has a 300-year-old history, as old as calculus itself, but its applications to physics and engineering have just begun [1]. Many physics and engineering systems have been found that they display fractional-order dynamics, such as electromagnetic waves [3], dielectric polarization[5],quantitative finance and diffusion wave [6] and so on. More recently, the chaotic behavior of fractional-order systems has been investigated [2-7]. Some examples of the systems include the fractional-order Liu system [8], the fractional-order Chen system [9], the fractional-order unified system[10] and so on. It has been well known that a hyper-chaotic attractor has more than one positive Lyapunov exponents which can increase the randomness of the corresponding system and improve the security of communication schemes by generating more complex dynamics. It is no doubt that high-dimensi-onal attractors have much wider application. Many scholars proofed the hyperchaotic behavior does exist in the fractional-order hyper-chaotic systems.

Inspired by above discussions, in this Letter, first of all, a new fractional-order hyperchaotic system is proposed and analyze the dynamics behavior of the system . On the other hand, we study the adaptive synchronization of the fractional-order hyperchaotic system based on feedback control principle. Corresponding theoretical analysis and numerical simulations are presented to verify the validity and feasibility of the proposed method.

\section{Preliminaries and notations}

There are several definitions of fractional derivatives [11], the commonly used definitions are Gunwald-Letnikov (GL), Remann-Liouville(RL), and Caputo(C) definitions.

The Grunwald-Letnikov(GL) derivative with fractional-order $q$ is given by

$$
{ }_{a}^{G L} D_{t}^{q} f(t)=\lim _{h \rightarrow 0} f_{h}^{(q)}(t)=\lim _{h \rightarrow 0} h^{-q} \sum_{i=0}^{\left[t-\frac{q}{h}\right]}(-1)^{i}\left(\begin{array}{l}
q \\
i
\end{array}\right) f(t-i h),
$$

where $[\bullet]$ means the integer part.

The Riemann-Liouville(RL) fractional derivatives are defined by

$$
J^{q} f(t)=\frac{1}{\Gamma(q)} \int_{0}^{t}(t-\tau) f(\tau) d \tau,
$$

where $\Gamma$ stands for Gamma function, 


$$
\Gamma(z)=\int_{0}^{\infty} t^{z-1} e^{-t} d t
$$

The Caputo-type fractional-order derivatives can be written as

$$
\frac{d^{\alpha} f(t)}{d t^{\alpha}}=J^{n-\alpha} \frac{d^{n} f(t)}{d t^{n}},
$$

where $n$ is the first integer which is not less than $\alpha$ and $J^{q}$ is the $q$-order

\section{Synchronization of fractional-system with unknown parameters based on feedback control}

\subsection{A novel fractional-order hyperchaotic system}

Before we study our fractional-order system, the mathematical description of the integer-order hyperchaotic system can be expressed as

$$
\left\{\begin{array}{l}
\dot{x}_{1}=a\left(x_{2}-x_{1}\right)+x_{4} \\
\dot{x}_{2}=x_{1} x_{3}-x_{2} \\
\dot{x}_{3}=b-x_{1} x_{2}-c x_{3} \\
\dot{x}_{4}=d x_{4}-x_{2} x_{3}
\end{array}\right.
$$

system (5) has a chaotic attractor when $a=5, b=16, c=1, d=-0.4$.

From the above equation, we improved the derivative operator in Eq.(5) to be with respect to a fractional-order $q$. So Eq.(5) can be converted to Eq.(6 )

$$
\left\{\begin{array}{l}
\frac{\mathrm{d}^{q_{1}} x_{1}}{\mathrm{~d} t^{q_{1}}}=a\left(x_{2}-x_{1}\right)+x_{4} \\
\frac{\mathrm{d}^{q_{2}} x_{2}}{\mathrm{~d} t^{q_{2}}}=x_{1} x_{3}-x_{2} \\
\frac{\mathrm{d}^{q_{3}} x_{3}}{\mathrm{~d} t^{q_{3}}}=b-x_{1} x_{2}-c x_{3} \\
\frac{\mathrm{d}^{q_{4}} x_{4}}{\mathrm{~d} t^{q_{4}}}=d x_{4}-x_{2} x_{3}
\end{array}\right.
$$

In the paper, we set order of fractional derivative $q=0.993$.

Figs.1 display the 2D phase portraits of the fractional-order hyperchaotic system, when $a=5, b=16, c=1, d=-0.4$.
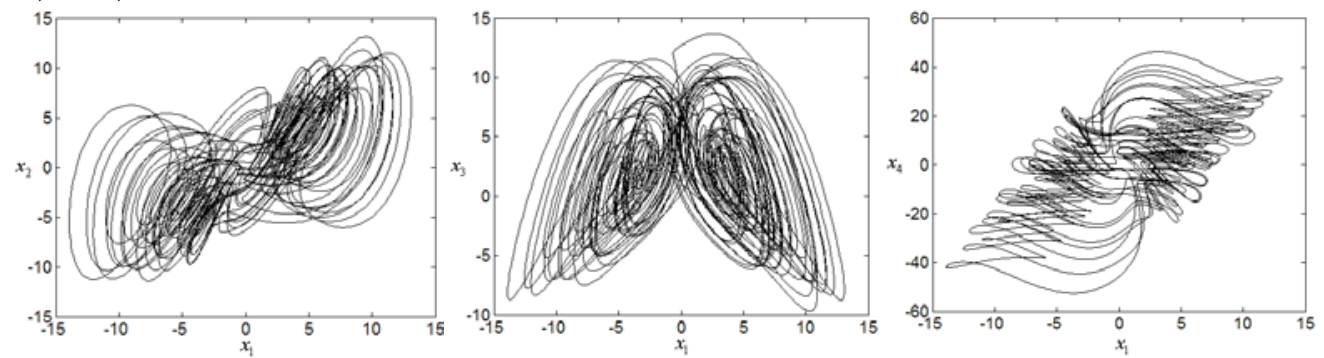

Figure 1. The chaotic attractor of system (6) with $a=5, b=16, c=1, d=-0.4$

\subsection{Synchronization of the hyperchaotic system with unknown parameters}

Consider the two different fractional-order chaotic systems given by

$$
\begin{aligned}
\frac{d^{q} x}{d t^{q}} & =A x+F(x), \\
\frac{d^{q} y}{d t^{q}} & =B Y+G(y),
\end{aligned}
$$

where $0<q \leq 1$ is the real order derivative, $x, y \in R^{n}$ are state variables of the system, $F(x), G(y) \in R^{n}$ are the nonlinear terms of the system (7) and system (8), respectively. $A, B$ are constant matrices. To 
achieve the synchronization of the above two different systems, via the feedback control, we add controller $u \in R^{n}$ in the system (8). Therefore, we can get the following equation

$$
\frac{d^{q} y}{d t^{q}}=B Y+g(y)+u \text {. }
$$

Our goal is to design the effective feedback controller $u$ which can synchronize the states of both the drive and response systems. The synchronization error variables are defined as $e=y-x$, then the corresponding error dynamics can be obtained as following

$$
\frac{d^{q} e}{d t^{q}}=B e+(B-A) x+G(y)-F(x)+u .
$$

Lemma 1[7] consider the autonomous system as following

$$
\frac{d^{q} x}{d t^{q}}=A x,
$$

where $x \in R^{n}, A \in R^{n \times n}$. when system (1) is asymptotically stable, if and only if $|\arg (\lambda)|>\frac{\alpha \pi}{2}$ is satisfied, where $\lambda$ is the eigenvalues of the matrix $A$.when system (1) is stable, if and only if $|\arg (\lambda)| \geq \frac{\alpha \pi}{2}$ is satisfied, where $\lambda$ is the eigenvalues of the matrix $A$.

Lemma 2 [7]. For the system (10), by choosing suit real symmetric positive matrix $P$, for state variable $x, J=x^{T} P \frac{d^{\alpha} x}{d t^{\alpha}} \leq 0$ is satisfied. In other words, the stats variable is asymptotically stable.

Theorem1. For the error system (13), if the controller is designed as below $u=(A-B) x+F(x)-G(y)+D G(x) e+c e$, where $D G(x)$ is the Jacobi of $G(x), C$ is a gain matrix to be designed later, the synchronization between systems (10) and (11) will be achieved for any initial values.

Proof : It follows from (10) and (11) that error dynamical system (13) can be written as

$$
\frac{d^{q} e}{d t^{q}}=B e+(B-A) x+G(y)-F(x)+(A-B) x+F(x)-G(y)+D G(x) e+c e
$$

According to the lemma 1 and 2, if $\left|\arg \left(\lambda_{i}(B+C)\right)>\frac{q \pi}{2}\right|, i=1,2 \ldots, n$ by choosing suit controller gain matrix control, then the fractional order chaotic system (5) and (6) can achieve to the synchronization.

In the following part, we realize the synchronization of system (6) via the adaptive synchronization method, and assume all parameters of the system are unknown. Define the drive system is system (6) and then the response system with controller $u=\left[u_{1}, u_{2}, u_{3}, u_{4}\right]^{T}$ can be described as following

$$
\left\{\begin{array}{l}
\frac{\mathrm{d}^{q} y_{1}}{\mathrm{~d} t^{q}}=\tilde{a}\left(y_{2}-y_{1}\right)+y_{4}+u_{1} \\
\frac{\mathrm{d}^{q} y_{2}}{\mathrm{~d} t^{q}}=y_{1} y_{3}-y_{2}+u_{2} \\
\frac{\mathrm{d}^{q} y_{3}}{\mathrm{~d} t^{q}}=\tilde{b}-y_{1} y_{2}-\tilde{c} y_{3}+u_{3} \\
\frac{\mathrm{d}^{q} y_{4}}{\mathrm{~d} t^{q}}=\tilde{d} y_{4}-y_{2} y_{3}+u_{4}
\end{array}\right.
$$

Our aim is investigate the synchronization of system (6) and (16). We define the error states $e_{1}=y_{1}-x_{1}, e_{2}=y_{2}-x_{2}, e_{3}=y_{3}-x_{3}, e_{4}=y_{4}-x_{4}, \tilde{a}, \tilde{b}, \tilde{c}, \tilde{d}$ are estimates of unknown parameters $a$,

$b, c, d$. The errors of unknown parameters are

$$
e_{a}=\tilde{a}-a, e_{b}=\tilde{b}-b, e_{c}=\tilde{c}-c, e_{d}=\tilde{d}-d,
$$

Theorem 2. The response system (16) can synchronize the drive system (6) globally and asymptotically as $t$ goes to infinite, if the controllers are taken as 


$$
\left\{\begin{array}{l}
u_{1}=\tilde{a}\left(x_{2}-x_{1}\right)-\tilde{a}\left(y_{2}-y_{1}\right)-e_{4}-e_{1} \\
u_{2}=x_{1} x_{3}-y_{1} y_{3} \\
u_{3}=y_{1} y_{2}-x_{1} x_{2}+\tilde{c} y_{3}-\tilde{c} x_{3}-e_{3} \\
u_{4}=y_{2} y_{3}-x_{2} x_{3}-\tilde{d} y_{4}+\tilde{d} x_{4}-e_{4}
\end{array}\right.
$$

and the adaptive laws of unknown parameters are taken as

$$
\left\{\begin{array}{l}
\frac{\mathrm{d}^{q} e_{a}}{\mathrm{~d} t^{q}}=\left(x_{1}-x_{2}\right) e_{1} \\
\frac{\mathrm{d}^{q} e_{b}}{\mathrm{~d} t^{q}}=-e_{3} \\
\frac{\mathrm{d}^{q} e_{c}}{\mathrm{~d} t^{q}}=x_{3} e_{3} \\
\frac{\mathrm{d}^{q} e_{d}}{\mathrm{~d} t^{q}}=-x_{4} e_{4}
\end{array}\right.
$$

Proof: From Eqs.(13) (16), the error dynamical system is

$$
\left\{\begin{array}{l}
\frac{\mathrm{d}^{q} e_{1}}{\mathrm{~d} t^{q}}=e_{a}\left(x_{2}-x_{1}\right)-e_{1} \\
\frac{\mathrm{d}^{q} e_{2}}{\mathrm{~d} t^{q}}=-e_{2} \\
\frac{\mathrm{d}^{q} e_{3}}{\mathrm{~d} t^{q}}=e_{b}-x_{3} e_{c}-e_{3} \\
\frac{\mathrm{d}^{q} e_{4}}{\mathrm{~d} t^{q}}=x_{4} e_{d}-e_{4}
\end{array}\right.
$$

According to the Lemma 2, the function candidate is taken as:

From Eq.(13-16)

$$
\begin{aligned}
J & =e_{1} \frac{d^{q} e_{1}}{d t^{q}}+e_{2} \frac{d^{q} e_{2}}{d t^{q}}+e_{3} \frac{d^{q} e_{3}}{d t^{q}}+e_{4} \frac{d^{q} e_{4}}{d t^{q}}+e_{a} \frac{d^{q} e_{a}}{d t^{q}}+e_{b} \frac{d^{q} e_{b}}{d t^{q}}+e_{c} \frac{d^{q} e_{c}}{d t^{q}}+e_{d} \frac{d^{q} e_{d}}{d t^{q}} \\
& =e_{1}\left(e_{a}\left(x_{2}-x_{1}\right)\right)+e_{2}\left(-e_{2}\right)+e_{3}\left(e_{b}-x_{3} e_{c}-e_{3}\right)+e_{4}\left(x_{4} e_{d}-e_{4}\right)+e_{a}\left(\left(x_{1}-x_{2}\right) e_{1}\right)+e_{b}\left(-e_{3}\right)+e_{c}\left(x_{3} e_{3}\right)+e_{d}\left(-x_{4} e_{4}\right) \\
& =-e_{1}{ }^{2}-e_{2}{ }^{2}-e_{3}{ }^{2}-e_{4}{ }^{2} .
\end{aligned}
$$

That is to say, the origin of error dynamical system (17) is asymptotically stable, the response system (13) can synchronize the drive system (6) globally and asymptotically.

For this numerical simulation, the unknown parameters of drive system (6) are set to $a=5, b=16$, $c=1, d=-0.4$. The initial condition of system(6) and (16) are $(3,6,2,7)$ and $(1,1,1,1)$, respectively. The state trajectories of the drive system and response system and error signals $e_{1}, e_{2}, e_{3}, e_{4}$ between systems (6) and (17), converge asymptotically zero , the estimated values of unknown parameters converge to $\tilde{a}=5, \tilde{b}=16, \tilde{c}=1, \tilde{d}=-0.4$, which are shown in Figures 2 and Figures 3.
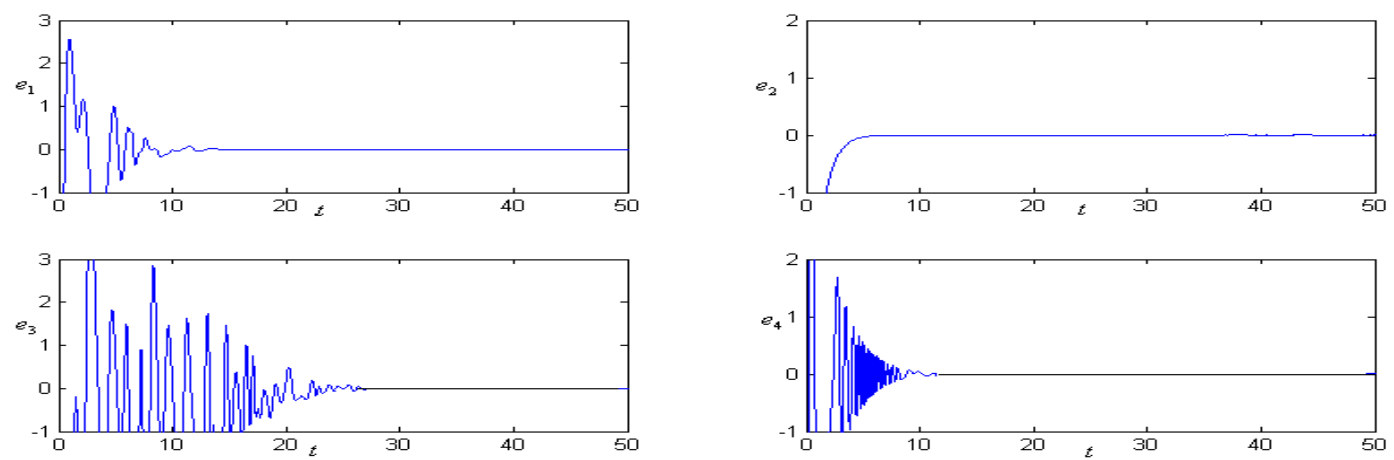

Figure.2. Synchronization errors between systems (6) and (17) with evolving time $t$ 

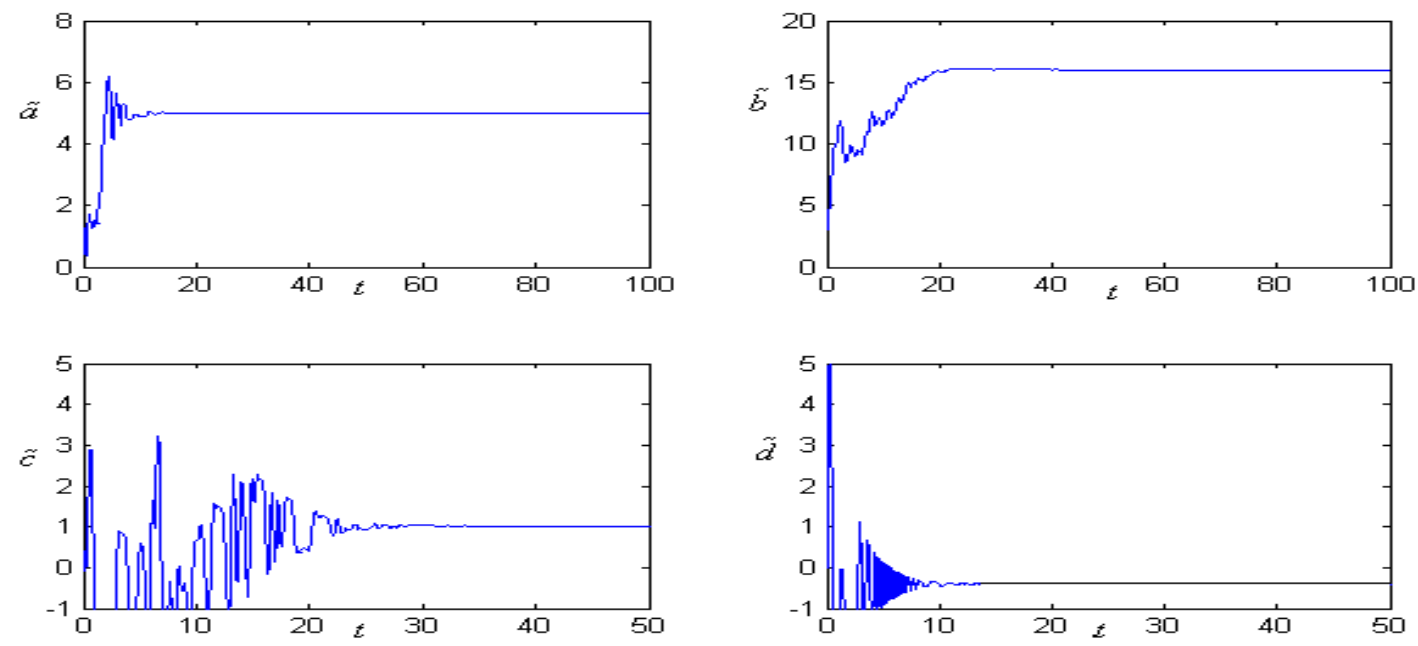

Figure.3. Identification curves of the unknown parameters of the response system (17)

\section{Conclusions}

In the article chaotic behaviors and the synchronization of a new fraction-order system have been studied. Furthermore, synchronization between two different pairs of hyperchaotic systems with uncertain parameters has been realized by feedback control. Based on the fractional stability theory, suitable adaptive synchronization controller and parameter identification rules of the unknown parameters are designed. Numerical simulations are carried out to demonstrate the effectiveness and feasibility of the controllers and identification rules.

\section{References}

[1] I. Podlubny, Fractional Differential Equations, Academic Press, New York,1999.

[2] K.Diethelm, The analysis of Fractional Differential Equations: An Application-Oriented Expos-ition Using Differential Operators of Caputo Type, Springer, Berlin, 2010.

[3] Westerlund S. Capacitor theory. IEEE Trans Dielect Electr Insul 1994;1(5):826-839.

[4] Hilfer, R.:Applications of Fractional Calculus in Physics. World Scientific, Singapore(2000).

[5] C.X. Liu, J.J. Lu, A novel fractional-order hyperchaotic system and its circuit realization. Int. J. Mod.Phys. B 24,(2010); 1299-1307.

[6] Jarad, F., Abdeljawad T., Baleanu, D.: Fractional variational principle with delay within Caputo derivatives. Rep. Math. Phys. 65(1)(2010)17-28.

[7] Bonnet C, Partington JR. Analysis of fractional delay systems of retarded and neutral type. Automatica 38(7) (2002):1133-1138.

[8] Ma J., Wang, Q.Y., Jin, W.Y., Xia,Y.F.: Control chaos in the Hindmars-Rose neuron by using intermittent feedback with one vrriable. Chin.Phys.Lett.25(2008),3582-3585.

[9] W.H.Deng,C.P.Li,Chaos synchronization of the fractional Lü system, PhysicaA353(2005)61-72.

[10] R.E.Mickens, Advances in the Applications of Nonstandard Finite Difference Schemes, World Scientific, Singapore,2005.

[11] Wang, X.Y., Song, J.M.: Synchronization of the fractional order hyperchaos Lorenz systems with activation feedback control. Commun. Nonlinear Sci. Numer. Simul. 14 (8),(2009);3351-3357. 